\title{
ERRATUM
}

\section{Erratum to: Withholding and withdrawal of life-sustaining treatments in low-middle- income versus high-income Asian countries and regions}

\author{
Jason Phua', Gavin M. Joynt ${ }^{2}$, Masaji Nishimura ${ }^{3}$, Yiyun Deng ${ }^{4}$, Sheila Nainan Myatra ${ }^{5}$, Yiong Huak Chan ${ }^{6}$, \\ Nguyen Gia Binh" ${ }^{7}$ Cheng Cheng Tan ${ }^{8}$, Mohammad Omar Faruq ${ }^{9}$, Yaseen M. Arabi ${ }^{10}$, Bambang Wahjuprajitno ${ }^{11}$, \\ Shih-Feng Liu ${ }^{12,13}$, Seyed Mohammad Reza Hashemian ${ }^{14}$, Waqar Kashif ${ }^{15}$, Dusit Staworn ${ }^{16}$, \\ Jose Emmanuel Palo ${ }^{17}$, Younsuck Koh ${ }^{18^{*}}$, ACME Study Investigators ${ }^{19}$ and Asian Critical Care Clinical Trials Group ${ }^{19}$
}

(C) 2016 Springer-Verlag Berlin Heidelberg and ESICM

\section{Erratum to: Intensive Care Med DOI 10.1007/s00134-016-4347-y}

Owing to an oversight in typesetting, the ACME Study Investigators and the Asian Critical Care Clinical Trials Group were initially omitted from the authorship. This error has now been rectified. The publisher apologizes for any inconvenience.

\section{Author details}

${ }^{1}$ Division of Respiratory and Critical Care Medicine, University Medicine Cluster, National University Hospital, National University Health System, Singapore, Singapore. ${ }^{2}$ Department of Anaesthesia and Intensive Care, The Chinese University of Hong Kong, Prince of Wales Hospital, Hong Kong, China. ${ }^{3}$ Emergency and Critical Care Medicine, University of Tokushima Graduate School, Tokushima, Japan. ${ }^{4}$ Intensive Care Unit, West China Hospital of Sichuan University, Chengdu, China. ${ }^{5}$ Department of Anaesthesia, Critical Care and Pain, Tata Memorial Hospital, Mumbai, India. ${ }^{6}$ Biostatistics Unit, Yong Loo Lin School of Medicine, National University of Singapore, Singapore,
Singapore. ${ }^{7}$ Intensive Care Department, Bach Mai Hospital, Hanoi, Vietnam. ${ }^{8}$ Department of Anaesthesia and Intensive Care, Sultanah Aminah Hospital, Johor Bahru, Malaysia. ${ }^{9}$ Department of Critical Care Medicine, BIRDEM General Hospital, Ibrahim Medical College, Dhaka, Bangladesh. ${ }^{10}$ King Saud Bin Abdulaziz University for Health Sciences and King Abdullah International Medical Research Center, Riyadh, Saudi Arabia. ${ }^{11}$ Department of Anesthesiology and Reanimation, Faculty of Medicine, University of Airlangga, Dr. Soetomo General Hospital, Surabaya, Indonesia. ${ }^{12}$ Division of Pulmonary and Critical Care Medicine, Department of Internal Medicine, Kaohsiung Chang Gung Memorial Hospital and Chang Gung University College of Medicine, Kaohsiung, Taiwan. ${ }^{13}$ Department of Respiratory Therapy, Kaohsiung Chang Gung Memorial Hospital and Chang Gung University College of Medicine, Kaohsiung, Taiwan. ${ }^{14}$ Chronic Respiratory Disease Research Center, National Research Institute of Tuberculosis and Lung Disease, Masih Daneshvari Hospital, Shahid Beheshti University of Medical Sciences, Tehran, Iran. ${ }^{15}$ Section of Nephrology, Pulmonary and Critical Care, Department of Medicine, Aga Khan University and Hospital, Karachi, Pakistan. ${ }^{16}$ Phramongkutklao Hospital, Bangkok, Thailand. ${ }^{17}$ The Medical City, Pasig City, Philippines. ${ }^{18}$ Department of Pulmonary and Critical Care Medicine, Asan Medical Center, University of Ulsan College of Medicine, 388-1 Pungnap Dong Songpa Ku, Seoul 138-736, South Korea. ${ }^{19} \mathrm{C} / \mathrm{o}$ Jason Phua, $1 \mathrm{E}$ Kent Ridge Road, Singapore 119228, Singapore.

Published online: 9 May 2016

The online version of the original article can be found under doi:10.1007/s00134-016-4347-y.

\footnotetext{
*Correspondence: yskoh@amc.seoul.kr

${ }^{18}$ Department of Pulmonary and Critical Care Medicine, Asan Medical Center, University of Ulsan College of Medicine, 388-1 Pungnap Dong Songpa Ku, Seoul 138-736, South Korea

Full author information is available at the end of the article
} 